\title{
Vólvulo de intestino medio: a propósito de dos casos
}

\author{
Middle intestine volvulus: on the purpose of two cases \\ Volvulus do intestino médio: com propósito de dois casos
}

L. Díaz ${ }^{1}$, M. Ormaechea², C. Juambeltz ${ }^{3}$

RESUMEN

La malrotación intestinal es un espectro de malformaciones, que incluye una gran variedad de alteraciones en el proceso de rotación y fijación del intestino. La ausencia completa de rotación intestinal, uno de los tipos de malrotación intestinal más frecuentes, puede presentarse como hallazgo asintomático o manifestarse clínicamente por un vólvulo del intestino medio. Sin embargo, incluso entre aquellos que se presentan con vólvulo del intestino medio, la clínica puede ser muy diferente, según el grado de isquemia y eventual necrosis intestinal.

Se presentan dos casos clínicos de malrotación intestinal con vólvulo del intestino medio con presentaciones, imágenes, tratamientos y evoluciones muy disimiles.

Se analizan los mismos a la luz de una revisión bibliográfica relevante al tema tratado, se sacan aprendizajes del manejo realizado y la evolución que presentaron, y se enfatizan los elementos de mayor jerarquía para optimizar el manejo de estos pacientes.

Palabras clave: Malrotación intestinal; Vólvulo de intestino medio.

\section{ABSTRACT}

Intestinal malrotation is a spectrum of malformations that includes a great variety of alterations in the rotation and fixation process of the intestines. The total absence of intestinal rotation, one of the most frequents types of intestinal malrotation could present as an asymptomatic find or appear clinically as a midgut volvulus. However, even those that appear as midgut volvulus, could show quite different signs and symptoms according to the degree of ischemic insult and possible intestinal necrosis.

Two clinical cases of intestinal malrotation with midgut volvulus with quite different presentations, images, treatments, and evolutions, are informed.

The two cases are analyzed under a relevant bibliographic revision, knowledge is derived from the carried-out management and evolution, and elements for the future optimization of management are underlined.

Keywords: Intestinal malrotation; Midgut volvulus.

RESUMO

A má rotação intestinal é um espectro de malformações, que inclui uma grande variedade de alterações no processo de rotação e fixação do intestino. A ausência completa de rotação intestinal, um dos tipos mais comuns de má rotação intestinal, pode se apresentar como um achado assintomático ou manifestar-se clinicamente como um volvo de intestino médio. Porém, mesmo entre aqueles que apresentam volvo de intestino médio, os sintomas podem ser muito diferentes, dependendo do grau de isquemia e eventual necrose intestinal.

Dois casos clinicos de má rotação intestinal com volvo de intestino médio são apresentados com apresentações, imagens, tratamentos e evoluções muito diferentes.
São analisados à luz de uma revisão bibliográfica pertinente ao tema em questão, lições aprendidas com o manejo realizado e a evolução que apresentaram, e os elementos de maior hierarquia são enfatizados para otimizar o manejo desses pacientes.

Palavras-chave: Má rotação intestinal; Volvulus do midgut.

\section{INTRODUCCIÓN}

La malrotación intestinal es, en realidad, un espectro de malformaciones, que incluye una gran variedad de alteraciones en el proceso de rotación y fijación del intestino. Las mismas van desde un ciego alto no acolado, hasta la ausencia completa de rotación ${ }^{(1)}$.

Su incidencia clínica es de 1 cada 6000 nacidos vivos, pero se ha informado como hallazgo asintomático en hasta el $0,2 \%$ de los estudios contrastados del tracto digestivo, y en 0.5 a $1 \%$ de las autopsias de población no seleccionada(2).

Un 60\% de los casos presentan otras malformaciones asociadas. Los pacientes portadores de gastrosquisis, onfalocele, y hernia diafragmática de Bochdaleck, presentan en forma constante, y como parte integral de su patología, una malrotación intestinal.

La ausencia completa de rotación intestinal, o malrotación intestinal de tipo IA según la clasificación de $\mathrm{H}$. Bill, tiene una presentación muy variable. La misma puede ir desde el vólvulo con isquemia y necrosis de todo el intestino medio, que se presentan con abdomen agudo, sepsis y shock, hasta pacientes completamente asintomáticos en los cuales el diagnóstico se realiza como un hallazgo al estudiarlos por otra causa. La conducta en los casos asintomáticos es controvertida. La opinión mayoritaria es que los menores de un año deben ser operados para evitar la complicación del vólvulo de intestino medio, cuya probabilidad es muy alta. En los pacientes mayores del año, la conducta es aún más discutida porque la frecuencia de esta complicación disminuye francamente ${ }^{(1)(3)}$.

El intestino medio se define como la porción del intestino que está irrigada por la arteria mesentérica superior y comprende el intestino delgado (yeyuno e ileon), el ciego, el colon ascendente y los dos tercios proximales del colon transverso. Normalmente el mesenterio del delgado se fija a la pared posterior 
del abdomen desde el ángulo duodeno-yeyunal o "ángulo de Treitz", localizado a la izquierda de la segunda vértebra lumbar, hasta el ciego, localizado en la fosa iliaca derecha. Esta base de implantación, es lo suficientemente larga como para impedir que el intestino rote sobre sí mismo(1). El mesocolon, además, se acola a la pared posterior del abdomen mediante la fascia de Toldt derecha y luego forma el mesocolon transverso entre ambos ángulos cólicos.

En los pacientes con ausencia completa de rotación intestinal, el mesenterio de todo el intestino medio (mesenterio común del delgado y el colon derecho) se fija a la pared posterior del abdomen, únicamente por una estrecha porción en torno al pedículo mesentérico superior, a los lados del cual se sitúan, el duodeno descendiendo por la derecha del abdomen y el colon transverso ascendiendo por la izquierda. Esta disposición permite que el intestino gire en torno al eje de la arteria mesentérica superior, determinando un vólvulo de todo el intestino medio, el cual, de acuerdo con lo apretado que resulte, puede o no determinar una obstrucción vascular aguda, con la consiguiente isquemia, necrosis, peritonitis, sepsis y shock. Por lo dicho, en ausencia de un diagnóstico temprano, el vólvulo de intestino medio está gravado de una alta mortalidad, y aún los que sobreviven tienen una alta morbilidad dada fundamentalmente por la insuficiencia intestinal, sus consecuencias y las complicaciones asociadas a su tratamiento. El diagnóstico temprano pasa por su sospecha, y por la realización de los estudios adecuados para confirmarlo o descartarlo, ante todo lactante con vómitos biliosos.

El procedimiento quirúrgico en los pacientes en los cuales el intestino se encuentra viable es la cirugía de Ladd. En los pacientes con necrosis, se hace necesaria la resección intestinal. Su longitud está directamente relacionada a la posibilidad de ocurrencia de una insuficiencia intestinal por intestino corto. En casos de isquemia sin necrosis consolidada, y a efectos de preservar la mayor cantidad de intestino posible, se acepta la devolvulación y reintroducción del intestino isquémico al abdomen, cerrándolo o dejándolo abierto (laparostomizado) mediante técnica de "open vacuum" o tipo "bolsa de Bogotá", para re explorarlo a las 24$48 \mathrm{hs}$., decidiendo entonces que sectores intestinales es necesario resecar y cuales pueden preservarse ${ }^{(4)(5)}$.

Se presentan dos casos de pacientes portadores de malrotación intestinal completa, complicados con vólvulo de intestino medio. Se analizan sus diferentes manifestaciones clínicas, procesos diagnósticos, estrategias terapéuticas y resultados clínicos.

\section{CASO 1}

Lactante de sexo femenino, de 68 dias de vida, con el antecedente de haber sido pretérmino de 27 semanas con $560 \mathrm{~g}$. de peso al nacer, y haber cursado un episodio interpretado como enterocolitis necrotizante a los 20 dias de vida, el cual evolucionó favorablemente con tratamiento médico exclusivo.

Internada desde el nacimiento, al momento del inicio de los sintomas se encontraba en cuidados intermedios, ventilando al aire, con alimentación enteral completa y bien tolerada, movilizando el intestino diariamente, y pesando $1400 \mathrm{~g}$.

Se consultó a la guardia de cirugía pediátrica por un cuadro de 6hs de evolución de distensión abdominal progresiva, de una entidad tal que, debido a la restricción respiratoria que generaba, la paciente debió someterse a asistencia ventilatoria mecánica de tipo invasivo, mediante intubación orotraqueal. Además, se había colocado una sonda nasogástrica por la que se obtuvo un residual gástrico de $30 \mathrm{ml}$ de leche. La última deposición había sido 10hs antes de la consulta quirúrgica y de características normales. La hemodinamia permanecia estable, y sin requerimientos de inotrópicos. En la evolución agregó una acidosis metabólica de tipo mixta.

Se solicitó una radiografía de abdomen que evidenció una gran distensión de asas intestinales, sin niveles hidroaéreos. No se observó neumoperitoneo, ni imágenes de neumatosis intestinal (Figura 1a y b).
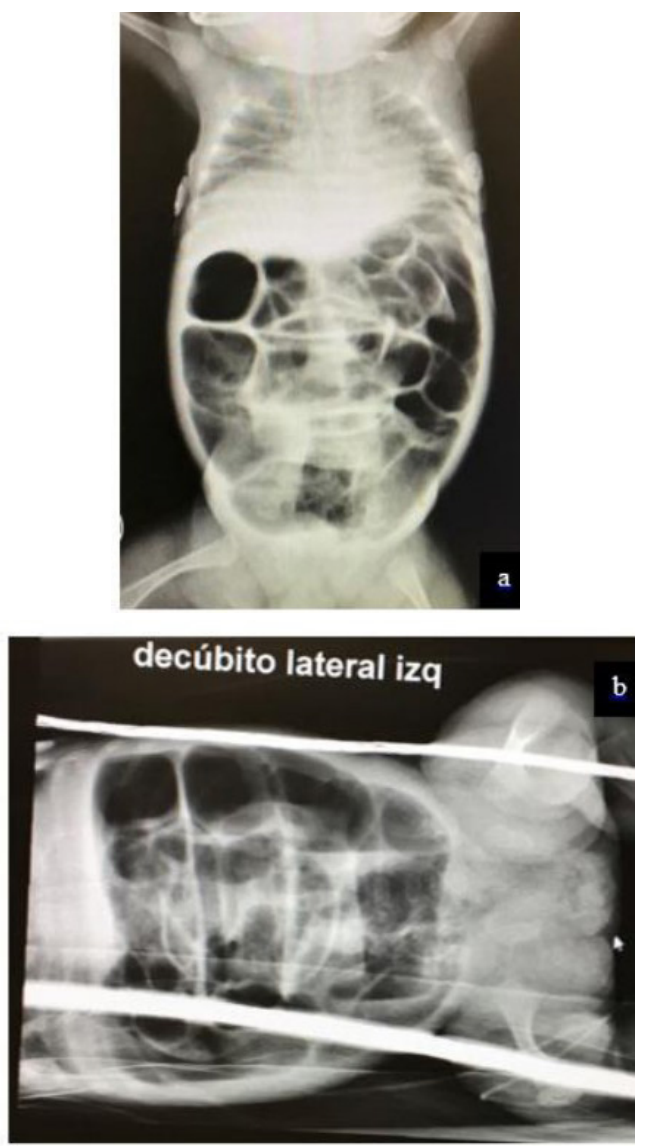

Figura 1. a y b) Rx de abdomen de la paciente que muestran una gran distensión gaseosa de todo el intestino, sin presencia de niveles hidroaéreos o neumoperitoneo.

Fuente: elaboración propia. 
Se realizó una ecografía de abdomen que informó la presencia de neumatosis portal y no logró observar los vasos mesentéricos debido a la gran interposición gaseosa.

Debido al aumento de la distensión abdominal, con progresivo deterioro respiratorio a pesar de la asistencia respiratoria mecánica, se decidió realizar una laparotomía exploradora de urgencia. Durante la cirugia se evidenció un vólvulo del intestino medio, con necrosis desde el ileon hasta el colon transverso y una perforación a nivel del íleon distal. Se realizó la resección de todo el sector necrótico, quedando indemnes los $45 \mathrm{~cm}$ proximales de intestino delgado y el colon desde el tercio distal del transverso. Se realizó una ileostomía proximal y se abocó el colon transverso a modo de fistula plana.

La paciente presentó una buena evolución en el postoperatorio inmediato, retomando la vía oral a los 7 dias. Debido al alto gasto por la ostomía y la imposibilidad de progresar en la alimentación enteral, se realizó la reconstrucción del tránsito digestivo a los 20 días. A pesar de esta reconstrucción temprana del tránsito digestivo, la paciente presentó una insuficiencia intestinal por intestino corto, que determinó la necesidad de alimentación parenteral prolongada. La rehabilitación intestinal llevó 1 año, luego del cual se pudo suspender la alimentación parenteral, logrando un crecimiento adecuado con aporte nutricional enteral exclusivo, y se otorgó el alta a domicilio. Actualmente la paciente se mantiene sin requerimientos de nutrición parenteral y presenta un buen ascenso ponderal en los controles.

\section{CASO 2}

Recién nacido de sexo masculino, de 5 dias de vida, que fue término, vigoroso, y adecuado para la edad gestacional, con un peso al nacer de $3000 \mathrm{~g}$.

El mismo se alimentó a pecho directo exclusivo, expulsó meconio a las $24 \mathrm{hs}$ de vida y recibió el alta a las $48 \mathrm{hs}$ de vida. En domicilio mantuvo un tránsito digestivo normal.

Consultó por un cuadro de $24 \mathrm{hs}$ de evolución de vómitos biliosos, acompañados de deposiciones con sangre roja escasa en dos oportunidades, y dolor abdominal cólico que calmaba espontáneamente. No refería distensión abdominal ni elementos de repercusión respiratoria, hemodinámica, o infecciosa o de compromiso del sensorio.

Al ingreso el paciente presentaba un buen estado general, en vigilia tranquila, ventilando espontáneamente al aire y con estabilidad hemodinámica. No impresionaba dolorido. Presentaba un abdomen no distendido, blando, depresible, y sin dolor a la palpación. Sin sectores empastados, ni cambios de coloración en la piel. Se colocó una sonda rectal obteniéndose escasa materia con sangre.

Los exámenes de laboratorio no presentaban alteraciones, destacando que no existía acidosis metabólica en la gasometría venosa.

Se solicitó radiografia de abdomen (Figura 2), que mostraba presencia de aire a nivel del estómago con escaso gas distal.

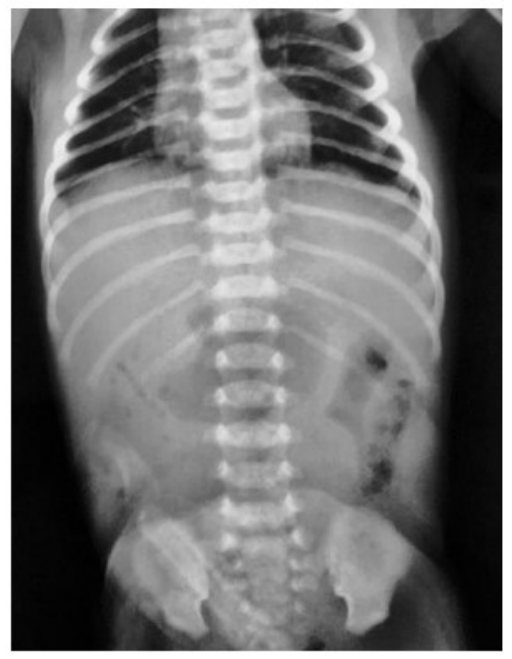

Figura 2. Rx de abdomen. Nótese la presencia de aire a nivel gástrico con la prácticamente ausencia de gases distales.

Fuente: elaboración propia.

En la evolución el paciente se mantuvo estable, sin episodios de dolor ni distensión abdominal, y movilizando el intestino normalmente, pero al intentar retomar la alimentación enteral, se obtuvieron residuales biliosos altos, por lo que se decidió suspender, nuevamente, el aporte enteral, y solicitar un estudio radiológico contrastado de esófago-gastroduodeno (Figura 3).

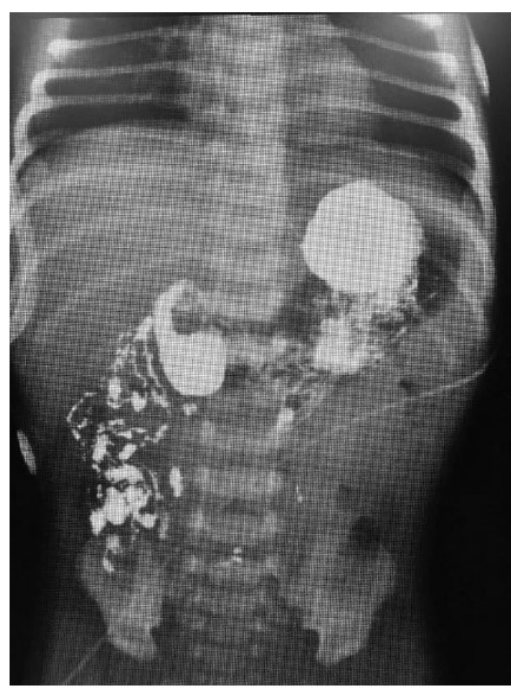

Figura 3. Estudio contrastado que muestra todo el intestino delgado a la derecha del abdomen con ausencia de llanta duodenal y ángulo duodeno-yeyunal, confirmando el diagnóstico de malrotación intestinal. Nótese el aspecto espiralado que adopta el contraste en el sector intestinal más proximal (insuficientemente jerarquizado durante la asistencia del paciente).

Fuente: elaboración propia. 
En el mismo no se observaron, ni la llanta duodenal y ni el ángulo duodeno-yeyunal, tal como se observa habitualmente a la izquierda de la columna. Por el contrario, el duodeno, el yeyuno y el resto del intestino delgado se encontraban a la derecha del abdomen, como ocurre en los pacientes malrotados.

Con el diagnóstico de malrotación intestinal sintomática se decidió realizar una laparotomía exploradora al día siguiente. En la exploración se constató un vólvulo del intestino medio, sin sufrimiento isquémico de este, pero con edema y adenitis en la raíz del mesenterio común (Figura 4). Presentaba además bridas de Ladd que contribuian a la obstrucción duodenal. Se realizó el procedimiento de Ladd, que comentaremos más adelante.

El paciente presentó una buena evolución postoperatoria, retomó la vía oral a las $48 \mathrm{hs}$ y recibió el alta a domicilio a los 5 dias. En el postoperatorio alejado, ha permanecido asintomático y presenta un crecimiento y desarrollo adecuados para la edad.

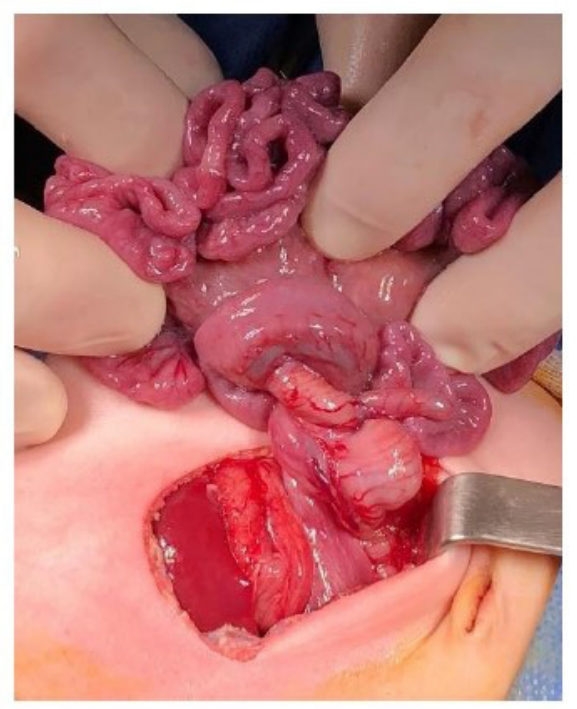

Figura 4. Imagen de la cirugia donde se observa el intestino medio volvulado, vital, sin isquemia.

Fuente: elaboración propia.

\section{DISCUSIÓN}

El vólvulo del intestino medio constituye la forma de presentación más frecuente de la malrotación intestinal. Éste puede manifestarse como un cuadro de extrema gravedad, vinculado a la isquemia y necrosis intestinal, o de forma oligo-sintomática con vómitos biliosos como única manifestación clínica.

El $75 \%$ de los pacientes con vólvulo de intestino medio se presentan antes del primer mes de vida, y un $15 \%$ más antes del primer año. Solo el $10 \%$ restante se presenta luego del año de vida, con franca disminución de la incidencia en relación con el aumento de la edad ${ }^{(1 .}$ $6,7)$

En su manifestación más grave se presenta como un cuadro agudo -en un lactante previamente sano o con patología previa, como nuestro primer paciente-, dado por dolor y distensión abdominal, acompañado de vómitos biliosos y en ocasiones deposiciones con sangre, secundarias a la isquemia mucosa, que rápidamente agrega acidosis metabólica y elementos de sepsis. En estos casos, el tiempo que se demore en llegar al diagnóstico y plantear la cirugía es clave en el pronóstico vital del intestino y cada minuto cuenta para minimizar la pérdida intestinal y mejorar el pronóstico vital y funcional del paciente.

En otros casos, como el de nuestro segundo paciente, el sintoma principal, y a veces único, pueden ser los vómitos biliosos o la imposibilidad de progresar la alimentación enteral. Son estos, casos que no llegan a instalar isquemia intestinal, y en los que predominan las manifestaciones de obstrucción duodenal incompleta. En ellos, solo un alto nivel de sospecha y una rápida realización de los estudios diagnósticos adecuados, nos va a permitir realizar un tratamiento quirúrgico urgente, antes de que se instale la isquemia intestinal, y permitiendo disminuir drásticamente la morbimortalidad de estos pacientes.

En cuanto a los estudios de imágenes, si bien la radiografía simple de abdomen puede tener cierta utilidad para plantear la sospecha clinica, cuando se encuentra distensión gastro duodenal -eventualmente con niveles hidroaéreos-, y ausencia de gases distales al duodeno comprometido en el pie del vólvulo, como se esboza en nuestro segundo paciente (no hay dilatación, pero si franca disminución de los gases distales), la misma es muy inespecifica y puede no aportar ningún elemento orientador, como ocurrió en nuestro primer paciente, que presentó una radiografia de abdomen con distensión gaseosa sin niveles hidroaéreos de todo el intestino, que incluso llegaba a simular un marco cólico normal, y en la que tampoco se apreció neumoperitoneo, a pesar de la perforación intestinal comprobada luego durante la cirugía.

El estudio por excelencia para el diagnóstico de la malrotación intestinal es el examen radiográfico contrastado del esófago-gastro-duodeno. En él se destaca la ausencia del arco duodenal característico, con el ángulo de Treitz a la izquierda de la columna, observándose en su lugar, como el contraste al salir del estómago se ubica rápidamente a la derecha de la columna, como vimos en el segundo paciente. Igualmente debemos recordar que, si bien menos frecuente, existen pacientes que presentan una rotación normal del duodeno, pero con una falta de rotación del colon, que también los pone en riesgo de sufrir un vólvulo del intestino medio.

En los casos en que la malrotación asocia un vólvulo de intestino medio, esta descripta la imagen en tirabuzón en las tomas de perfil, que corresponden 
al sector volvulado. Destacamos que, en el estudio contrastado de nuestro segundo paciente, se observa un aspecto espiralado del sector más proximal de intestino, que debió conducir al planteo de vólvulo y que fue pasado por alto.

A pesar de ser este, de los casos presentados, el que tuvo la mejor evolución, analizado en este momento vemos que la interpretación de este estudio fue insuficiente. Es una enseñanza para futuros casos, la importancia que se debe dar a una imagen de este tipo. Aún en ausencia de elementos clínicos que sugieran isquemia intestinal, esta imagen debe hacer plantear la posibilidad de vólvulo y debe llevar a una cirugia de urgencia a continuación de su identificación y el vaciado del estómago mediante la aspiración por sonda nasogástrica.

En lo que refiere a la ecografía de abdomen, cuando la malrotación intestinal es una posibilidad diagnóstica, la misma debe ser realizada con doppler de los vasos mesentéricos. Como siempre es importante la experiencia del colega que la realice, tanto en pediatría como en el uso del doppler. Se puede visualizar una inversión en la relación de la vena mesentérica superior con respecto a la arteria mesentérica superior, ubicándose la primera a la izquierda de la segunda, o en aproximadamente el $20 \%$ de los casos, la vena en posición ventral a la arteria. La sensibilidad de la malposición vascular es de un 53 a un $87 \%$, para el diagnóstico de malrotación intestinal.

El signo ecográfico del remolino, descrito por Pracros en 1992, es característico del vólvulo del intestino medio(8), y esta imagen es dada por la vena mesentérica superior y sus afluentes, junto a la grasa mesentérica y las asas intestinales girando en torno de la arteria mesentérica superior ${ }^{(9)}$.

Una de las limitaciones de la ecografía en los casos de vólvulo del intestino medio, puede ser la interposición gaseosa que dificulta la adecuada visualización de los vasos mesentéricos, como ocurrió en nuestro primer caso.

Debido a la tendencia a utilizar cada vez con mayor frecuencia estudios de imágenes, y a la mejoría en su definición, la neumatosis portal (gas a nivel del flujo porta) es un hallazgo cada vez más frecuente. Si bien se ha visto que constituye un signo inespecifico, que puede verse en múltiples fenómenos inflamatorios del intestino y la cavidad peritoneal e incluso sin causa detectable, y que por tanto su interpretación depende del contexto clínico, en el caso de nuestro segundo paciente, y acompañando a un cuadro grave, de dolory distensión abdominal, con acidosis mixta, debe apoyar el planteo de lesión intestinal isquémica con pasaje de gases desde la luz intestinal a la circulación portal.

En los casos de rotaciones incompletas el estudio contrastado del colon por enema evidencia la posición final que adoptó el ciego confirmando la malrotación.

Se debe tener siempre presente que, estos estudios se realizarán en la medida que el cuadro clínico del paciente lo permita, pero en pacientes con alta sospecha de vólvulo de intestino medio, o incluso de presentar un abdomen quirúrgico, aún con dudas sobre su etiologia, no deben retrasar el tratamiento quirúrgico, el cual es perfectamente admisible que sea indicado fundamentado exclusivamente por la clínica.

Respecto a los estudios de laboratorio, estos pacientes pueden presentar acidosis metabólica, por isquemia intestinal y aumento de los parámetros infecciosos como ocurrió en el primer paciente, que presentó elevación de los leucocitos ehiperplaquetosis.

El tratamiento de los pacientes malrotados con vólvulo del intestino medio siempre es quirúrgico y de urgencia.

En pacientes con vitalidad intestinal conservada, se realiza el procedimiento descripto por Ladd en 1936. cuando comunicó el tratamiento de 20 pacientes con malrotación intestinal ${ }^{(14)}$.

El mismo consta de los siguientes pasos: evisceración completa del intestino, devolvulación (dado que habitualmente el intestino rota en sentido horario, esta debe realizarse en sentido antihorario -viendo al paciente desde los pies-), sección de las bridas de Ladd si existen y rectificación del duodeno, apertura amplia de la raíz del mesenterio común ensanchando su base, apendicectomía (controversial en el momento actual, pero descripta en la técnica original) y por último reintroducción del intestino al abdomen dejando el delgado a la derecha de la columna y el colon a la izquierda, con el ciego a nivel del flanco izquierdo.

$\mathrm{Si}$ hay sectores claramente necróticos, deben resecarse. A efectos de minimizar la exéresis intestinal y la producción de un sindrome de intestino corto, pueden mantenerse sectores isquémicos 0 de vitalidad dudosa tras la devolvulación, para luego realizar un "second look" y definir en ese momento que debe resecarse y que preservarse. Esto puede permitir realizar resecciones más acotadas ${ }^{(5)}$.

Este concepto ha sido utilizado de forma exitosa para la enterocolitis necrotizante desde 1986(4). En 1992 Hoffman MA et al. publicaron el caso de un neonato con vólvulo del intestino medio e isquemia severa desde el ligamento de Treitz hasta el colon transverso, en el que se logró preservar la mayoría del intestino mediante esta forma de manejo ${ }^{(15)}$.

En nuestro primer paciente el intestino medio se encontraba claramente necrosado. El único sector de viabilidad dudosa era la primera asa delgada, la cual se preservó y presentó una buena evolución. Luego de la resección se midieron $45 \mathrm{~cm}$ de intestino delgado remanente, más el tercio distal de colon transverso en adelante. Destacamos que la imposibilidad de 
preservar la válvula ileocecal, como ocurrió en este paciente, genera mayores dificultades durante el proceso de rehabilitación intestinal. En el segundo paciente, dado que no presentaba elementos de sufrimiento isquémico, no fue necesaria la realización de resecciones intestinales, y se realizó el procedimiento de Ladd sin incidentes.

El abordaje puede ser abierto o laparoscópico ${ }^{(10,11)}$. En estos casos se utilizó el abordaje abierto, que es el más utilizado a nivel mundial.

Si bien el abordaje laparoscópico se ha convertido en algo frecuente en la edad pediátrica, e incluso en varias patologias neonatales, aún son pocos los trabajos sobre su utilización para la malrotación intestinal con vólvulo en el periodo neonatal ${ }^{(12)}$. En 1995 se publicó el primer caso del uso de la laparoscopía para devolvular un intestino medio seguido por un procedimiento de Ladd y en 1996 el abordaje laparoscópico se describió en el manejo de un caso de malrotación sin vólvulo.

Debido a que existen condiciones propias del cuadro clínico que generan un muy dificultoso manejo laparoscópico, con riesgos específicos que superan a los conocidos beneficios del abordaje laparoscópico frente al abierto, es que este se reserva para pacientes muy seleccionados. Un estudio realizado por Martínez Ferro en 2006 plantea que el abordaje laparoscópico es seguro en pacientes seleccionados. El mismo propone como candidatos a dicho abordaje aquellos con hemodinamia estable, sin alteraciones en la ventilación, sin perforación intestinal, sin distensión importante, sin presencia de asa fija en la radiografia, y sin signos ecográficos de isquemia intestinal severa (engrosamiento de la pared intestinal y/o interrupción completa y abrupta del flujo mesentérico)(13). Otros estudios más recientes refieren que el uso de la laparoscopia constituye un abordaje seguro para estos pacientes disminuyendo el tiempo de internación, dolor postoperatorio y mejores resultados estéticos ${ }^{(13)}$.

El pronóstico de estos pacientes es variable. Un paciente operado sin isquemia tiene una mortalidad prácticamente nula, pero esta puede elevarse hasta el $50 \%{ }^{(1)}$ entre aquellos que requieren resecciones amplias del intestino medio. La evolución a cuadros graves puede ser más o menos rápida, dependiendo de la forma de presentación. Sin embargo, el grado de sospecha diagnóstica y a la rapidez y calidad de la evaluación por parte del cirujano, pueden tener una incidencia muy importante en el pronóstico de muchos de estos niños. El optimizar estos elementos, será fundamental para minimizar la ocurrencia de sepsis y shock asociadas a la isquemia y necrosis intestinal, así como la necesidad de resecciones intestinales extensas, insuficiencia intestinal, desnutrición, y las múltiples complicaciones vinculadas a internaciones y dependencia de nutrición parenteral en forma prolongada.

\section{EN SUMA}

Hemos visto dos neonatos portadores de una malrotación intestinal complicada con un vólvulo del intestino medio. Esto nos ha permitido apreciar las diferentes presentaciones clínicas e imagenológicas que pueden tener estos pacientes y nos ha permitido aprender del manejo realizado, esperando que todo esto contribuya a optimizar el manejo de estos pacientes a futuro.

\section{REFERENCIAS}

1. Cannizzaro C, Martinez Ferro M, Chattás G. Fetoneonatología Quirúrgica. 1. Buenos Aires: Ediciones Journal; 2018

2. Holcomb GW, Murphy JP, Peter SS. Ashcraft's Pediatric Surgery. 7 ed. Elsevier; 2019 .

3. Malek MM, Burd RS. The optimal management of malrotation diagnosed after infancy: a decision analysis. Am J Surg. 2006:191(1):45-51

4. Weber TR, Lewis JE. The role of second-look laparotomy in necrotizing enterocolitis. J Pediatr Surg. 1986;21(4):323-5.

5. Jan I, Ziaullah M, Obaid L, Hassan M, Shehhi M. Planned Second look laparotomy in neonatal volvulus - A safe approach for bowel salvage. Pak J Med Sci. 2018:34(2):508-10.

6. Durkin ET, Lund DP, Shaaban AF, Schurr MJ, Weber SM. Agerelated differences in diagnosis and morbidity of intestinal malrotation. J Am Coll Surg. 2008;206(4):658-63.

7. Berrocal T, Gayá F, de Pablo L. Aspectos embriológicos, clínicos y radiológicos de la malrotación intestinal. Radiología. 2005:47(5):237-51.

8. Rokade ML, Yamgar S, Tawri D. Ultrasound "Whirlpool Sign" for Midgut Volvulus. Journal of Medical Ultrasound. 2011;19(1):246.

9. Hamidi H, Obaidy Y, Maroof S. Intestinal malrotation and midgut volvulus. Radiol Case Rep. 2016;11(3):271-4

10. Hagendoorn J, Vieira-Travassos D, van der Zee D. Laparoscopic treatment of intestinal malrotation in neonates and infants: retrospective study. Surgical Endoscopy. 2011;25(1):217-20.

11. Palanivelu C, Rangarajan M, Roshan A, Jani K. Intestinal Malrotation with Midgut Volvulus Presenting as Acute Abdomen in Children: Value of Diagnostic and Therapeutic Laparoscopy. Journal of Laparoendoscopic \& Advanced Surgical Techniques. 2007:17(4):490-2.

12. Ferrero L, Ahmed Y, Philippe P, Reinberg O, Lacreuse I, Schneider A, et al. Intestinal Malrotation and Volvulus in Neonates: Laparoscopy Versus Open Laparotomy. J Laparoendosc Adv Surg Tech A. 2017:27(3):318-21.

13. Martinez-Ferro $\mathbf{M}$, Bignon $\mathbf{H}$, Figueroa $\mathbf{M}$. Operación de Ladd laparoscópica en el recién nacido. Cir Pediatr. 2006;19:182-4.

14. Disorders of intestinal rotation and fixation. In: Grosfeld J, O'Neill J, Coran A, Fonkalsrud E (Ed.) Pediatric Surgery. Philadelphia: Elsevier; 2006. Cap 84. v.2. p.1342-135

15. Hoffman MA, Johnson CL, Moore T, Pearl RH. Management of catastrophic neonatal midgut volvulus with a silo and second-look laparotomy. J Pediatr Surg. 1992;27(10):1336-9. 


\section{Nota de contribución:}

La Dra. Díaz elaboró el artículo.

El Dr. Ormaecha realizó la corrección.

El Dr. Juambeltz realizó la supervisión.

\section{Nota del Editor:}

El editor a cargo del presente manuscrito fue Patricia Braga.

Recibido: 21/08/2020

Aceptado: 17/02/2021 